\title{
Nucleon Spectroscopy with CLAS
}

\author{
Steffen Strauch ${ }^{1, \star}$ for the CLAS Collaboration \\ ${ }^{1}$ University of South Carolina, Columbia, SC 29208
}

\begin{abstract}
Meson photoproduction is an important tool in the study of nucleon resonances. The spectrum of broad and overlapping nucleon excitations can be greatly clarified by use of polarization observables. The $N^{*}$ program at Jefferson Lab with the CEBAF Large Acceptance Spectrometer (CLAS) includes experimental studies with linearly- and circularly-polarized tagged-photon beams, longitudinally- and transversely-polarized nucleon targets, and recoil polarizations. Selected results from these experimental studies will be presented.
\end{abstract}

\section{Introduction}

The nature of QCD confinement continues to provide an ongoing challenge to our understanding of non-perturbative QCD. Studying the nucleon spectrum provides one approach to learn more about this unique feature since the location and properties of excited states reflect the dynamics and relevant degrees-of-freedom within hadrons. Symmetric quark models with three independent quark degrees of freedom predict an overabundance of higher-lying excited states compared to what has been observed until now [1]. Lattice QCD calculations predict states with the same quantum numbers as constituent quark models with an underlying $S U(6) \times O(3)$ symmetry and find a counting of levels that is consistent with those models [2]. Quark-diquark models with a 'frozen' diquark predict fewer states. To clarify the nucleon resonance spectrum with the help of new experimental data is essential to inform studies of QCD.

Nucleon resonances have hadronic decay channels and are typically short-lived. They have large widths and overlap in the resonance spectrum. Identifying resonances and extracting resonance information in partial-wave analyses (PWA) of experimental data is challenging and requires not only unpolarized cross-section data, but also phase information from polarization observables. The photoproduction of a single pion, for example, is fully described by four complex amplitudes. It requires a minimum of eight carefully chosen observables at each energy and angle to determine the amplitudes of the reaction completely [3]. The photoproduction of two mesons requires even more observables [4]. Most present resonance information originate from $\pi N$ reactions. Some resonances, however, couple weakly to the $\pi N$ channel and multiple final states need to be investigated to fully explore the resonance spectrum.

The CLAS Collaboration has performed a series of photoproduction experiments off nucleons as part of the $N^{*}$ program at the Thomas Jefferson National Accelerator Facility to provide the needed

^e-mail: strauch@sc.edu 
data. These measurements cover a variety of final states including single pseudoscalar-mesons, vectormesons, double-pions, and hyperons. The observables include cross sections, single- and doublepolarization observables. The incident bremsstrahlung photon beams were energy-tagged [5] and either unpolarized, circularly, or linearly polarized. A central component of this program are experiments off polarized protons [6-10] and neutrons [11]. These experiments were made possible by the construction of the frozen-spin polarized butanol (FROST) [12] and hydrogen-deuteride (HDice) [13] targets. Final-state particles were detected in the CEBAF Large Acceptance Spectrometer (CLAS) [14] over a wide angular range, excluding the very beam-forward and backward directions. A toroidal magnetic field and packages of drift chambers allowed for the determination of the particle momentum. Time-of-flight detectors helped in the particle identification. The CLAS detector has in the meantime been replaced by CLAS12. Few selected results from these CLAS photoproduction experiments off polarized nucleons are discussed in the following.

\section{CLAS Results}

\subsection{Pion Photoproduction}

The double-polarization observable $E$ in the $\gamma p \rightarrow \pi^{+} n$ reaction can be determined in an experiment that measures the helicity asymmetry of circularly-polarized photons incident on longitudinallypolarized protons. The CLAS collaboration measured this process in the center-of-mass energy range from $W=1.25 \mathrm{GeV}$ to $W=2.23 \mathrm{GeV}$ [15]. Figure 1 shows angular distributions of the data for selected $W$ bins. The upper panels show that previous PWA results describe the new data where the PWA have been well constrained by previous data at low energies, however they fail to describe the data at higher energies. The data have subsequently been included in new analyses of the SAID, Jülich-Bonn, and Bonn-Gatchina groups. The groups achieved an overall satisfying description of the data, lower panels of Fig. 1, that was obtained by a series of smaller updates of nucleon resonance parameters. The process led to an improved consistency of helicity amplitudes among the various analyses. One particularly interesting result is strengthened evidence for the poorly known $\Delta(2200) \frac{7}{2}^{-}$resonance in improving the Bonn-Gatchina fit at the highest energies [16]. The mass of the $\Delta(2200) \frac{7}{2}^{-}$resonance is significantly higher than the mass of its parity partner $\Delta(1950) \frac{7}{2}^{+}$, which is the lowest-mass $\Delta^{*}$ resonance with spin-parity $J^{P}=\frac{7}{2}^{+}$. This finding has relevance for the hypothesis of chiral-symmetry restoration at high mass.

Other single-pion photoproduction channels are also being studied. For example, results from $\gamma p \rightarrow \pi^{0} p$ off transversally polarized protons have been obtained in an analysis of the target $T$ and beam-target $F$ polarization observables [20]. The preliminary results agree with world data in the range of overlap at lower energies, but provide new information up to center-of-mass energies of $W \approx 2.5 \mathrm{GeV}$.

The lack of free-neutron targets makes experiments off the neutron difficult and results for observables off the proton dominate the data bases. However, information from both isospin channels are necessary to completely specify the amplitudes of the reaction. Novel data have been obtained in quasielastic photoproduction reactions off polarized neutrons from the CLAS g14 experiments. Figure 2 shows CLAS results for the polarization observable $E$ in the $\gamma n(p) \rightarrow \pi^{-} p(p)$ reaction [21] as blue data points. The data are compared to updated fits of the SAID and Bonn Gatchina analyses. Three selected $W$ bins show also previous fits and demonstrate how much the new data helped constraining those new analyses. The inclusion of these results in the new analyses has resulted in revised $\gamma n N^{*}$ couplings and, in the case of the $N(2190) \frac{7}{2}^{-}$, to convergence among different partial-wave analyses. The $\gamma n N^{*}$ couplings are sensitive to the dynamical process of $N^{*}$ excitation and provide important guides to nucleon structure models. 


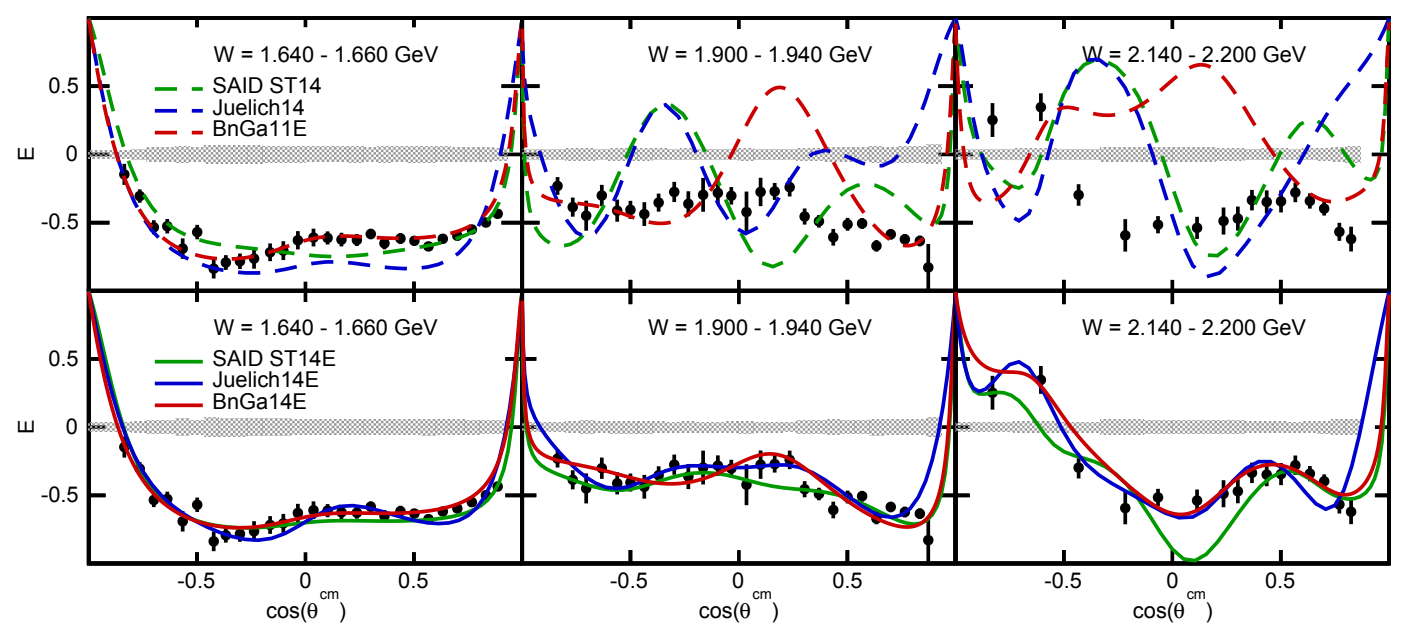

Figure 1. CLAS data for the beam-target polarization observable $E$ in the $\vec{\gamma} \vec{p} \rightarrow \pi^{+} n$ reaction for selected $W$ bins. The shaded bands indicate systematic uncertainties. The curves are results of partial-wave analyses before (upper panels) and after (lower panels) including the new CLAS data. The previous analyses are from SAID ST14 [17], Jülich14 [18], and BnGa11E [19]. Figure from Ref. [15].

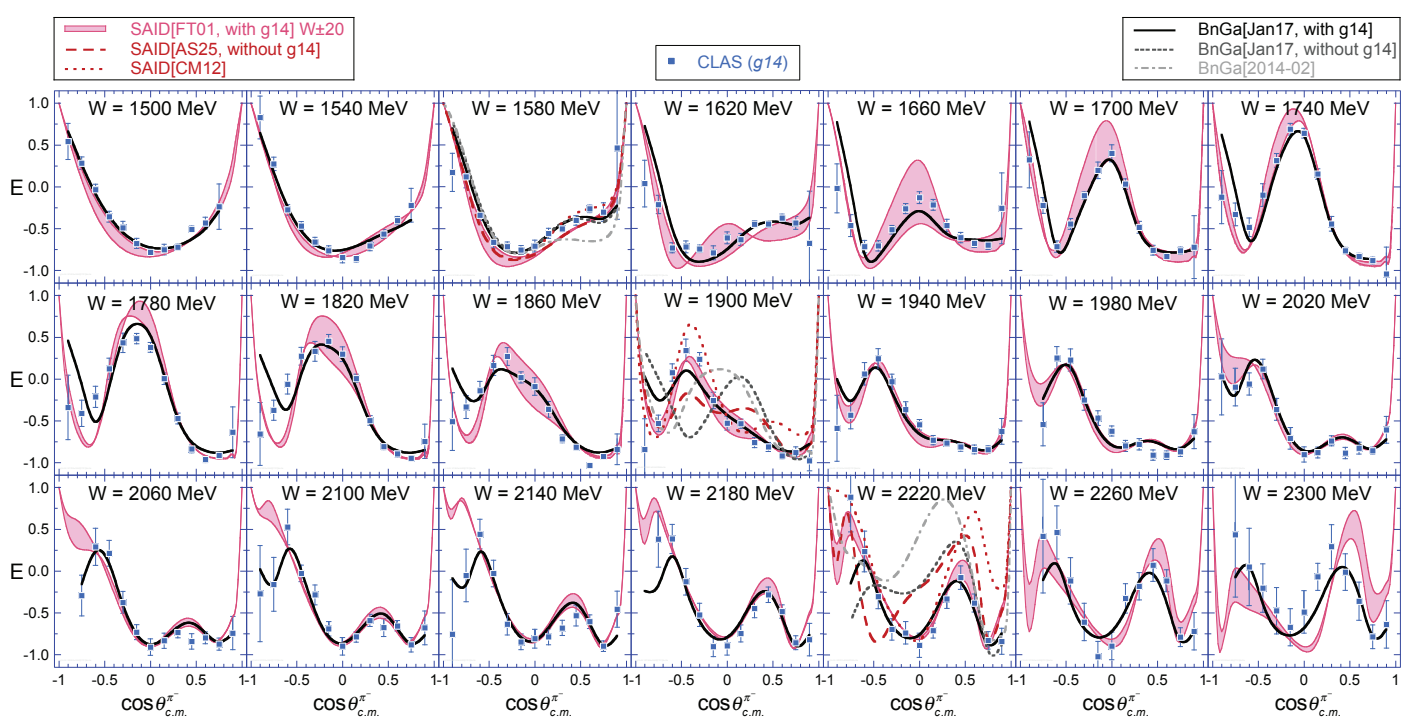

Figure 2. CLAS data for the beam-target polarization observable $E$ in the $\vec{\gamma} \vec{n} \rightarrow \pi^{-} p$ reaction (blue data points). SAID and Bonn-Gatchina analyses that include these data are shown as red bands and solid black lines, respectively. Figure from Ref. [21]. 


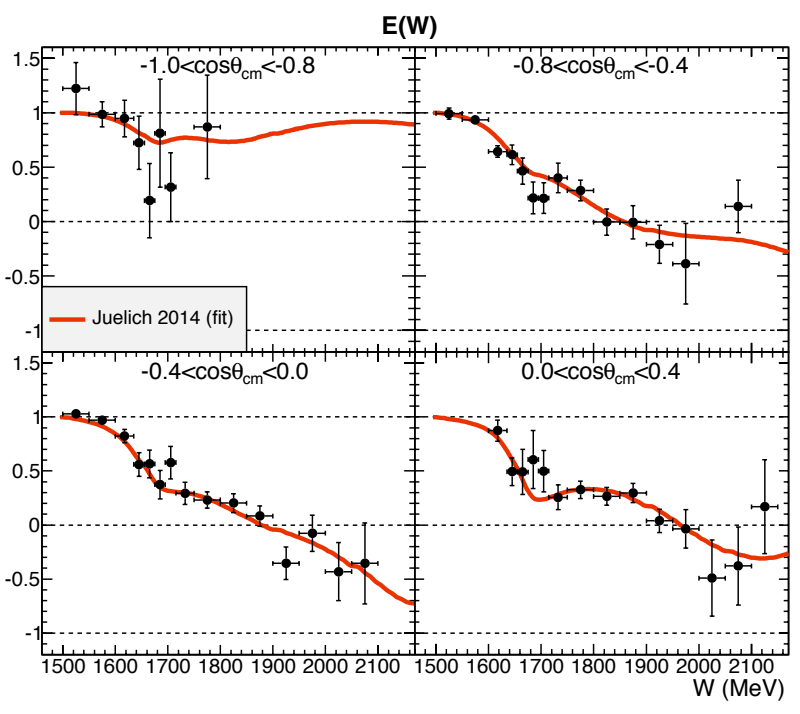

Figure 3. Energy dependence of the helicity asymmetry $E$ in the $\vec{\gamma} \vec{p} \rightarrow \eta p$ reaction for various angular bins. The red curves show calculations of the Jülich-Bonn dynamical coupled-channel model that were fit to the CLAS data (black points). Figure from Ref. [22].

\subsection{Eta Photoproduction}

The $\eta$ meson is an isospin-zero particle and $\eta$ photoproduction selectively excites isospin- $\frac{1}{2}$ resonances of the nucleon resonance spectrum. The CLAS collaboration has measured the polarization observable $E$ in the $\gamma p \rightarrow \eta p$ reaction from threshold to $W=2.15 \mathrm{GeV}$ [22]. Figure 3 shows the energy dependence of $E$. In the threshold region $E$ approaches unity. The data were fit with the Jülich-Bonn dynamical coupled-channel model (red curves). The fit describes the data quite well without the need for an additional narrow resonance near $1.68 \mathrm{GeV}$, which was previously suggested [22].

\subsection{Hyperon Photoproduction}

The analysis of the angular distribution of the parity-violating weak decay of hyperons allows for the reconstruction of the hyperon recoil polarization in the reactions $\gamma p \rightarrow K^{+} \Lambda$ and $\gamma p \rightarrow K^{+} \Sigma^{0}$. This gives access to additional recoil-polarization observables. CLAS measurements of the $\Lambda$ induced polarization $P[23,24]$ as well as the polarization-transfers $C_{x}$ and $C_{z}$ from circularly-polarized photons to the recoiling hyperons [25] were critical input to a coupled-channel analysis by the Bonn-Gatchina group $[26,27]$. In particular, the analysis found further evidence for the, at the time, poorly known $N(1900) \frac{3}{2}^{+}$resonance. This resonance is predicted by symmetric three-quark models, but is not expected to exist in earlier quark-diquark models. New hyperon-photoproduction data with linearlypolarized photons off an unpolarized proton target have been obtained up to $W \approx 2.2 \mathrm{GeV}$. Together with the recoil polarization of the hyperon, this gives access to five polarization observables: $\Sigma, P, T$, $O_{x}$, and $O_{z}$. Figure 4 shows as an example the beam-recoil polarization observable $O_{z}$ for the $K^{+} \Lambda$ channel as a function of center-of-mass energy $W$ for eight angular bins of $\cos \theta_{K}$ of the kaon's polar angle. The data are compared with predictions of the ANL-Osaka (red bands) coupled-channel model and the 2014 Bonn-Gatchina partial-wave analysis (green bands). None of those describe the new data well. A refit of the $\mathrm{BnGa}$ analysis to the data gives an excellent description. This refit includes two new nucleon resonances with spin and parity of $J^{P}=\frac{3}{2}^{+}$and $\frac{5}{2}^{+}$. However, no strong evidence for their existence can be derived from the data. 


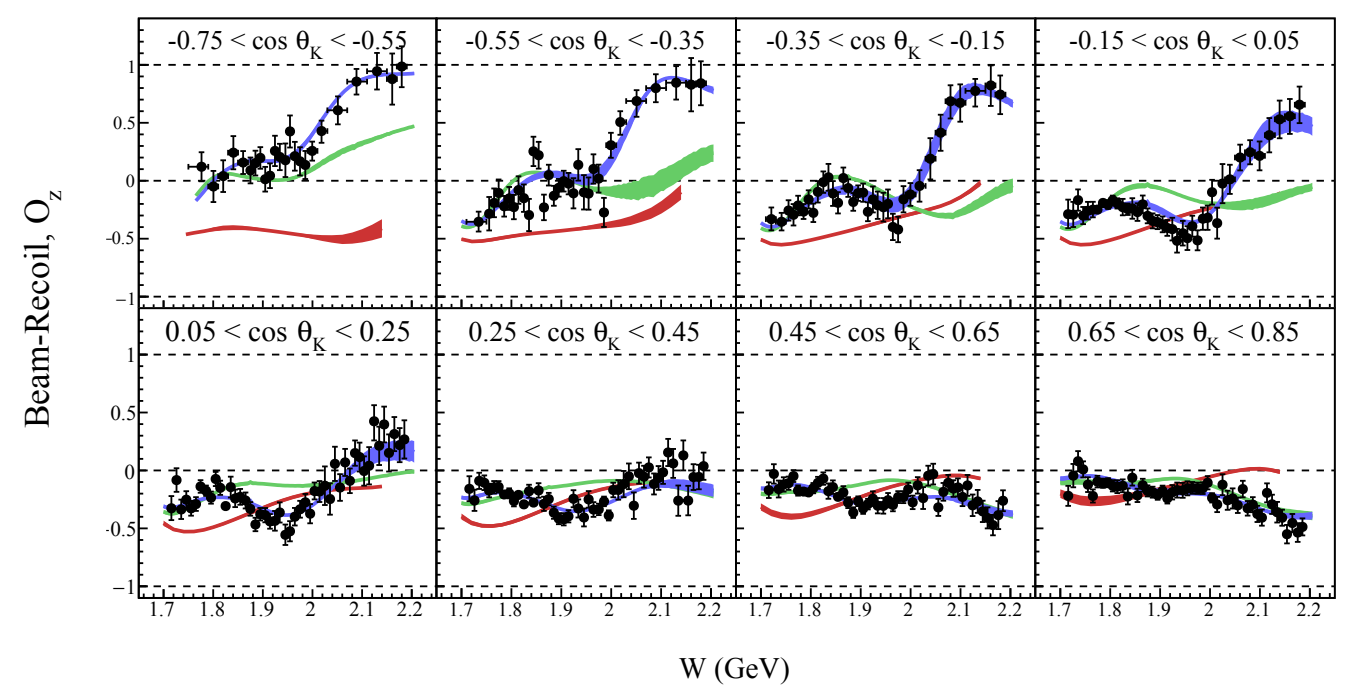

Figure 4. CLAS data of the beam-recoil double-polarization observable $O_{z}$ in the $\vec{\gamma} p \rightarrow K \vec{\Lambda}$ reaction. The red and green curves show predictions from the ANL-Osaka coupled-channel and Bonn-Gatchina partical-wave analyses, respectively. The blue curves show a Bonn-Gatchina refit to the new data. Figure from Ref. [28].

\subsection{Double-Pion Photoproduction}

Higher-lying nucleon resonances above about $1.6 \mathrm{GeV}$ have significant branching ratios for $N^{*} \rightarrow \pi \Delta$ or $N^{*} \rightarrow \rho N$ that lead to $\pi \pi N$ final states. This reaction channel and the study of the sequential decays contain potentially rich information about the nucleon-resonance spectrum. Many polarization observables are accessible in two-pion photoproduction off the nucleon [4]. The CLAS Collaboration was first to study the beam-helicity asymmetry $I^{\odot}$ for the $\gamma p \rightarrow \pi^{+} \pi^{-} p$ reaction. The measurements covered energies between $W=1.35$ and $2.30 \mathrm{GeV}$ [29]. Many more polarization observables were measured with the FROST experiment: the target observables $P_{i}$, beam-target observables $P_{i}^{\odot}$ for circularly polarized photons, and beam-target observables $P_{i}^{s, c}$ for linearly polarized photons; $i=x, y$ are for reactions off transversely- and $i=z$ off longitudinally-polarized nucleons. Figure 5 shows as one example preliminary results for observable $P_{x}$ as a function of the azimuthal angle $\phi^{*}$ in seven $\cos \theta_{C M}$ bins for $W \approx 1600 \mathrm{MeV}$. See Fig. 6 for the definition of the angles. This observable is accessible in measurements with unpolarized photons off transversally-polarized protons. The observable is an odd function of the azimuthal angle $\phi^{*}$. This is highlighted by a fit of a sum of $\sin n \phi^{*}$ terms to the data (red curves). The data are compared to bin-averaged results of a calculation by Fix and Arenhövel [30] based on an effective Lagrangian approach (green curves). The model results resemble key features of the preliminary data, but seem to be off in phase. Once finalized, the data will strongly constrain models. 


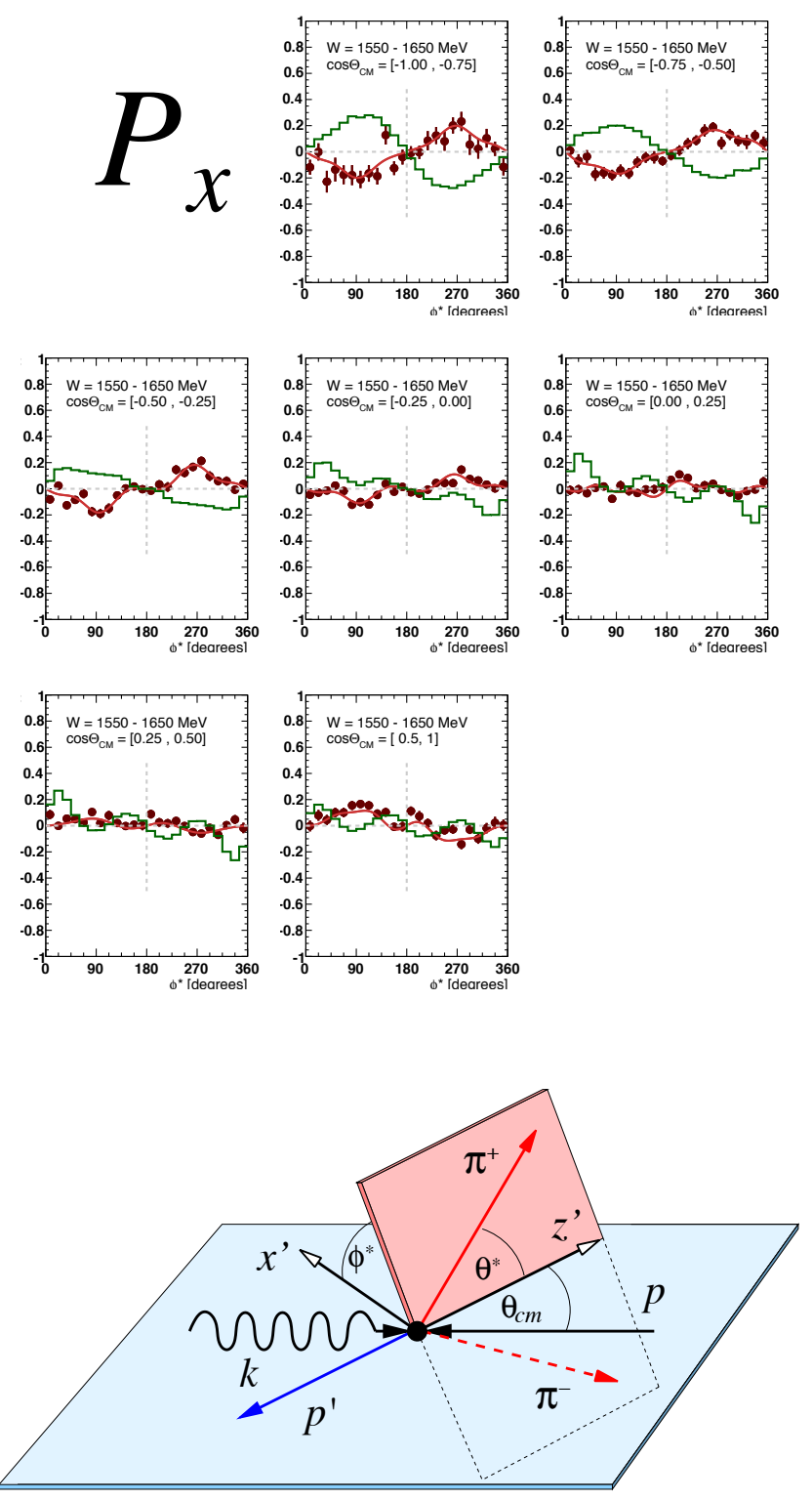

Figure 5. Preliminary $\phi^{*}$ angular distributions for the target-polarization observable $P_{x}$ in the $\gamma p \rightarrow \pi^{+} \pi^{-} p$ reaction for a center-of-mass energy of $W=1550-1650 \mathrm{MeV}$ and seven bins in $\cos \theta_{C M}$ from CLAS. Angles are defined in Fig. 6. The green cuves show bin-averaged results of a model calculation from A. Fix [30]. The red curves are fits of a sum of $\sin n \phi^{*}$ terms to the data. Figure from [31].

Figure 6. Cartoon of the $\gamma p \rightarrow \pi^{+} \pi^{-} p$ reaction with definitions of kinematic variables.

\section{Conclusion}

The Particle Data Group routinely updates its compilation of $N$ - and $\Delta$-resonance information. Major input came from analyses of CLAS data. CLAS data continue to make an impact as new results for polarization observables in photoproduction reactions off protons and neutrons become available. The new data will contribute to complete or nearly-complete experiments, and will challenge previously 
poorly constrained models. It is very likely that these data will have a tremendous impact on the understanding of baryon resonances and may provide evidence for new states found in coupled-channel analyses.

This work was supported in parts by the U.S. National Science Foundation: NSF PHY-1505615.

\section{References}

[1] V. Credé, W. Roberts, Rept. Prog. Phys. 76, 076301 (2013)

[2] R.G. Edwards, J.J. Dudek, D.G. Richards, S.J. Wallace, Phys. Rev. D84, 074508 (2011), 1104.5152

[3] W.T. Chiang, F. Tabakin, Phys. Rev. C55, 2054 (1997)

[4] W. Roberts, T. Oed, Phys.Rev. C71, 055201 (2005), nucl-th/0410012

[5] D. Sober et al., Nucl. Instrum. Meth. A 440, 263 (2000)

[6] Jefferson Lab Experiment E02-112, "Search for Missing Nucleon Resonances in Hyperon Photoproduction”, P. Eugenio, F. Klein, and L. Todor, spokespersons

[7] Jefferson Lab Experiment E03-105, "Pion Photoproduction from a Polarized Target", N. Benmouna, W. Briscoe, G. O'Rielly, I. Strakovsky, S. Strauch, spokespersons

[8] Jefferson Lab experiment E04-102, "Helicity Structure of Pion Photoproduction”, D. Crabb, M. Khandaker, and D. Sober, spokespersons

[9] Jefferson Lab experiment E05-012, "Measurement of polarization observables in $\eta$ photoproduction with CLAS", M. Dugger and E. Pasyuk, spokespersons

[10] Jefferson Lab Experiment E06-013, "Measurement of $\pi^{+} \pi^{-}$Photoproduction in DoublePolarization Experiments using CLAS”, M. Bellis, V. Credé, S. Strauch, spokespersons

[11] Jefferson Lab Experiment E06-101, " $\mathrm{N}^{*}$ Resonances in Pseudoscalar-meson photo-production from Polarized Neutrons in $\vec{H} \cdot \vec{D}$ and a complete determination of the $\gamma n \rightarrow K^{0} \Lambda$ amplitude",

F. Klein and A.M. Sandorfi, spkespersons

[12] C. Keith, J. Brock, C. Carlin, S. Comer, D. Kashy, J. McAndrew, D. Meekins, E. Pasyuk, J. Pierce, M. Seely, Nucl. Instrum. Meth. A 684, 27 (2012)

[13] M.M. Lowry et al., Nucl. Instrum. Meth. A 815, 31 (2016)

[14] B.A. Mecking et al., Nucl. Instrum. Meth. A 503, 513 (2003)

[15] S. Strauch et al. (CLAS), Phys. Lett. B750, 53 (2015), 1503.05163

[16] A.V. Anisovich, V. Burkert, J. Hartmann, E. Klempt, V.A. Nikonov, E. Pasyuk, A.V. Sarantsev, S. Strauch, U. Thoma, Phys. Lett. B766, 357 (2017), 1503.05774

[17] R.L. Workman, M.W. Paris, W.J. Briscoe, I.I. Strakovsky, Phys. Rev. C86, 015202 (2012)

[18] D. Rönchen, M. Döring, F. Huang, H. Haberzettl, J. Haidenbauer, C. Hanhart, S. Krewald, U.G. Meißner, K. Nakayama, Eur. Phys. J. A50, 101 (2014), 1401.0634

[19] A.V. Anisovich, R. Beck, E. Klempt, V.A. Nikonov, A.V. Sarantsev, U. Thoma, Eur. Phys. J. A48, 15 (2012), 1112.4937

[20] H. Jiang, Ph.D. thesis, University of South Carolina (2017)

[21] D. Ho et al. (CLAS), Phys. Rev. Lett. 118, 242002 (2017), 1705.04713

[22] I. Senderovich et al. (CLAS), Phys. Lett. B755, 64 (2016), 1507.00325

[23] J.W.C. McNabb et al. (CLAS), Phys. Rev. C69, 042201 (2004), nucl-ex/0305028

[24] M.E. McCracken et al. (CLAS), Phys. Rev. C81, 025201 (2010), 0912 . 4274

[25] R.K. Bradford et al. (CLAS), Phys. Rev. C75, 035205 (2007), nucl-ex/0611034

[26] A.V. Sarantsev, V.A. Nikonov, A.V. Anisovich, E. Klempt, U. Thoma, Eur. Phys. J. A25, 441 (2005), hep-ex/0506011 
[27] V.A. Nikonov, A.V. Anisovich, E. Klempt, A.V. Sarantsev, U. Thoma, Phys. Lett. B662, 245 (2008), 0707. 3600

[28] C.A. Paterson et al. (CLAS), Phys. Rev. C93, 065201 (2016), 1603.06492

[29] S. Strauch et al. (CLAS Collaboration), Phys. Rev. Lett. 95, 162003 (2005), hep-ex/0508002

[30] A. Fix, H. Arenhövel, Eur.Phys.J. A25, 115 (2005), nucl-th/0503042

[31] L.A. Net, Ph.D. thesis, University of South Carolina (2017) 